\title{
A TERAPIA MULTIFAMILIAR NO TRATAMENTO DA DEPENDÊNCIA QUÍMICA: UM ESTUDO RETROSPECTIVO DE SEIS ANOS ${ }^{1}$
}

\author{
Susana M. Sastre Seadi* \\ Margareth da Silva Oliveira**
}

\section{RESUMO}

Introdução: A inclusão de intervenções focadas na família, através da terapia unifamiliar, ou da terapia multifamiliar (TGMF) vem crescendo como uma forma de enfrentar um problema tão grave e complexo como é a dependência química. Objetivos: Investigar e avaliar fatores associados à adesão ao tratamento multifamiliar no tratamento de dependentes químicos hospitalizados. Método: A pesquisa é um estudo transversal retrospectivo, com uma amostra de 672 famílias participantes da TGMF durante o período de seis anos (de março 1997 a julho de 2003). Foi realizado um estudo dos fatores sociodemográficos como idade, sexo, tempo de uso da substância e quanto ao tipo de droga mais prevalente e investigada a associação entre o grau de parentesco do familiar participante do programa e a adesão ao tratamento multifamiliar. Foram pesquisados 672 prontuários de sujeitos que estiveram internados e ingressaram no programa de tratamento multifamiliar e os relatos das sessōes descritos pelo terapeuta coordenador do grupo. Resultados: Há associação entre a participação da família e adesão ao tratamento. A participação de dois ou mais familiares repercute na adesão. Discussão: Os resultados sugerem que inclusão de um número maior de familiares poderá repercutir em uma maior adesão ao tratamento.

* Mestre em Psicologia Clínica pela Pontifícia Universidade Católica do Rio Grande do Sul (PUCRS).

** Doutora em Ciências pela Universidade Federal de São Paulo (UNFESP); Orientadora e coordenadora do Grupo de pesquisa "Intervençōes Cognitivas e Comportamentos Dependentes” do Programa de Pós-Graduação em Psicologia Clínica da Pontifícia Universidade Católica do Rio Grande do Sul (PUCRS). 
Palavras-chave: transtornos relacionados ao uso de substâncias; terapia familiar; pacientes desistentes do tratamento.

\begin{abstract}
MULTI-FAMILY THERAPY AND DRUG ADDICTION: A SIX-YEAR RETROSPECTIVE STUDY

Introduction: the systematic inclusion of interventions focused on the family through unifamily and/or through multi-family group therapy (MFGT) has been growing as a way to face such a serious and complex problem as drug addiction. Objective: To investigate and evaluate factors associated with the joining of drug addicted patients to a multi-family treatment. Method: the research is a cross-sectional retrospective study, with a sample of 672 families taking part in MFGT for six years (from March, 1997 to July, 2003). A study about sociodemographic factors such as age, gender, time and predominant type of substance used was made, which also looked into the relation between which family members took part in the program and the number of patients joining the multi-family treatment. 672 inpatient files were researched. The reports of the meetings described by the coordinating therapist of the group were also researched. Results: a relation between family participation and patients joining the treatment was found. Discussion: the results suggest that the inclusion of a higher number of family members can promote greater treatment commitment in drug addicted patients.
\end{abstract}

Keywords: substance-related disorders; family therapy; patient dropout.

\title{
INTRODUÇÃO
}

A dependência química é um fenômeno de extrema relevância em termos de saúde pública, é multifatorial e sua complexidade exige que o tratamento implique em múltiplas abordagens terapêuticas (Silva, 2001). A terapia unifamiliar e/ou multifamiliar é inserida nesse contexto, intervindo nas famílias em plena crise.

Vários trabalhos na literatura (De Micheli \& Formigoni, 2001; Liddle et al., 2001; Meyers, Apodaca, Ficker \& Slenisck, 2002; Stanton \& Todd, 1987; Steinglasss, Bennett, Wolin \& Reiss, 1987) têm abordado a dependência de drogas como um fenômeno que afeta não somente o usuário, mas também seu sistema familiar, enfatizando assim a importância do estudo do funcionamento relacional dessas famílias.

Para cada indivíduo envolvido com álcool e/ou outras drogas, estima-se que 4 a 5 pessoas, incluindo cônjuges, companheiros, filhos e pais serão direta ou indiretamente afetados. Um episódio de embriaguez e intoxicação pode repercutir em um importante comprometimento das relações familiares refletindo-se diretamente nas crianças (Halpern, 2001). 
$\mathrm{Na}$ maior parte dos casos, as instituições e os terapeutas recebem o dependente químico com a incumbência de entregá-lo "curado" à família, que efetivamente não é percebida como parte integrante do problema e, portanto, do processo de mudança. A passagem para a epistemologia ecossistêmica redimensiona essa visão, ampliando o foco, impedindo de fixá-lo no paciente identificado (Laranjeira, Jungerman \& Dunn, 1998; Lopes \& Seadi, 2002).

Na prática clínica já está estabelecido que a abordagem familiar é importante no tratamento de farmacodependentes em geral, mas, no tratamento de adolescentes usuários de drogas, a terapia familiar parece ser fundamental (Scivoletto \& Andrade, 2001).

Em trabalho de revisão (Schenker \& Minayo, 2004) sobre a importância da família no tratamento do uso abusivo de substâncias psicoativas, foram encontrados vários estudos empíricos cujos resultados atestam a efetividade dos tratamentos nos quais a família em si é o objeto de intervenção, com melhores resultados, se comparados àqueles centrados no paciente identificado. Porém, nesse estudo, as autoras verificaram que entre muitos profissionais de saúde não existe ainda o reconhecimento destas evidências, pois não são incorporadas nos contextos onde exercem suas práticas clínicas. As razões talvez residam em um escasso acesso a estas investigações, sinalizando uma lacuna, um distanciamento entre a pesquisa e o exercício da clínica.

$\mathrm{Na}$ terapia de família utiliza-se a terapia multifamiliar, uma técnica que se caracteriza por oportunizar um contexto em que os padróes de relacionamento intrafamiliares e os relacionais das famílias com a comunidade podem ser percebidos (Jancin, 2003; Thorngren \& Kleist, 2002).

Outra característica da abordagem multifamiliar é possibilitar a cada membro do grupo ver os demais em interação, isto é, passar da compreensão particular à compreensão do outro, ampliando a percepção tanto das dificuldades quanto das formas de solucioná-las. $\mathrm{O}$ atendimento multifamiliar oportuniza às famílias repensarem os seus conceitos e incluírem-se no projeto de mudança.

Unem-se à terapia de família sistêmica os fatores terapêuticos do processo de grupo e estudos confirmam que esta associação resulta em um ambiente fértil para explorar comportamentos individuais no contexto dos relacionamentos interpessoais. Desencadeia a ampliação da consciência de grupo e de comunidade e, assim, do suporte social necessário para que sejam feitas as mudanças de comportamento desejadas (Ravazzola, Barilari \& Mazieres, 1997).

A maioria dos familiares espera aprender a lidar com o dependente e receber orientação profissional. E há evidências da eficácia da terapia breve e grupal no sentido de adequar condutas, contribuindo para a melhora das relações e organizaçôes do contexto familiar em dependência química (Figlie \& Pillion, 2001). 
A abordagem multifamiliar como uma intervenção breve na dependência química não entende os problemas das famílias como uma doença e sim como padrões relacionais disfuncionais e é focada nos recursos e habilidades que as famílias possuem para resolver os seus problemas. A revisão de literatura aponta para a importância da intervenção breve em problemas relacionados ao abuso e dependência de substâncias psicoativas. Afirma-se que existem evidências consistentes para recomendar a adoção de intervenções breves em diferentes contextos de tratamento como uma conduta básica pela sua efetividade comprovada, por ser um recurso economicamente viável, oportunizando o atendimento de milhares de pessoas envolvidas com problemas associados ao uso de substâncias psicoativas no Brasil (Marques \& Furtado, 2004; Marques \& Ribeiro, 2003).

A participação das mães, segundo estudo de Contel e Villas-Boas (1999), realizado no grupo de apoio multifamiliar em um hospital-dia psiquiátrico, repercute na adesão ao tratamento, corroborando outros achados da literatura que, por sua vez, ratificam a presença das mães como a mais constante. Estes autores encontraram relação do tempo de permanência de um paciente no tratamento com maior número de sessões frequentadas pelo familiar. As mulheres, especialmente as mães, foram a presença mais constante, respondendo por $80 \%$ de presença em cada grupo e marido e mulher raramente frequentavam conjuntamente os grupos.

A terapia multifamiliar é uma técnica particularmente útil e aplicável para abusadores e suas famílias. Esse tipo de terapia pode ser utilizado em qualquer contexto de tratamento da dependência química, mas é mais efetivo nos tratamentos em regime hospitalar porque é quando as famílias estão mais disponíveis e acessíveis (Sugar, 1986).

Tendo em vista a alta prevalência dos transtornos por uso de substância psicoativa e o papel importante da família no processo terapêutico destes sujeitos, bem como o número reduzido de estudos que abordam este tema, o objetivo desta pesquisa foi investigar os fatores que repercutem ou não na adesão da família ao tratamento multifamiliar.

\section{MÉTodo}

\section{DELINEAMENTO}

Trata-se de um estudo retrospectivo de levantamento de dados, coletados ao longo de seis anos, com base em prontuários de atendimento multifamiliar. As 
substâncias utilizadas pelos pacientes identificados foram: álcool, cocaína, maconha e outras substâncias. A nicotina não constou das drogas pesquisadas.

\section{PARTICIPANTES}

Foram pesquisados 672 sujeitos hospitalizados em uma unidade de desintoxicação de uma clínica psiquiátrica, especializada no tratamento de dependência química, cujos familiares participaram no mínimo de um encontro do grupo multifamiliar, no período compreendido entre março de 1997 e julho de 2003.

\section{INSTRUMENTOS}

O instrumento adotado foi uma ficha com dados sociodemográficos e informações quanto ao consumo de substâncias psicoativas e à frequência dos familiares às sessões.

\section{PROCEDIMENTO}

O procedimento adotado inicialmente foi contatar a clínica onde seria realizada a pesquisa e buscar junto à sua direção geral autorização para pesquisar e utilizar os dados neste estudo. Esta pesquisa foi aprovada pelo Comitê de Ética em Pesquisa da PUCRS.

\section{Programa de TeRapia Multifamiliar}

A abordagem multifamiliar foi inserida no Programa para que o tratamento fosse extensivo à família.

O processo terapêutico era desenvolvido em 6 (seis) sessōes visando contemplar as principais etapas do tratamento hospitalar: a fase pós-desintoxicação, $o$ início das abordagens individuais e o imediatamente após a alta hospitalar e início da ressocialização.

O grupo multifamiliar era aberto com duração ilimitada quanto à sua existência, mas limitado para cada família, pois todo o processo terapêutico tinha a duração de seis sessões. Os grupos eram de final em aberto, com famílias saindo 
deles e outras neles ingressando. As sessões multifamiliares eram semanais e tinham a duração de uma hora.

Paciente designado, identificado, reconhecido. Esta expressão é utilizada para designar aquela pessoa cuja situação provocou uma demanda de tratamento por parte de um ou vários membros da família e mesmo daqueles profissionais obrigados a intervir na realidade (Miermont \& Molina-Loza, 1994).

O paciente identificado deveria ter concluído a etapa de desintoxicação que dura em média uma semana; ter ingressado no Programa de tratamento com duração de mais ou menos 21 dias. O paciente podia participar das sessões multifamiliares somente acompanhado de no mínimo um familiar e/ou de alguém do sistema significativo. Não havia restrição quanto aos tipos de configurações familiares, grau de instrução, grau de parentesco ou idade para a participação no grupo.

Não era permitida a participação do paciente identificado e/ou seus familiares e/ou pessoas com vínculos significativos se estivessem intoxicados; o paciente não poderia estar desligado administrativamente do programa ou ter havido uma interrupção do tratamento independentemente da sua causa. Não era permitida a participação do paciente identificado sozinho, nem do familiar desacompanhado do paciente. Estava vedada a participação do paciente identificado ou seus familiares e/ou pessoas da rede social se apresentassem um funcionamento psicótico.

Havia contraindicação para a participação do núcleo familiar cujo paciente identificado e/ou seus familiares fossem pessoas de reconhecimento público (como por exemplo políticos, autoridades, artistas), evitando-se submetê-las à exposição e sabendo-se que este critério é válido para os grupos em geral e não somente para grupos multifamiliares.

Quanto ao número de participantes, variava desde sessões com duas famílias até sessões denominadas numerosas, comportando várias famílias com a presença de dezenas de pessoas.

As sessões começavam com a apresentação e o acolhimento das famílias iniciantes. E sempre que famílias estavam na $6^{a}$ sessão era oferecido um espaço para um depoimento, avaliação e despedida do grupo, ao final da sessão. Para as famílias que estavam enfrentando o tratamento do paciente identificado pela primeira vez, o sofrimento incluía também a dificuldade para realizarem a internação que em alguns casos iniciara de forma compulsória. Para esse grupo os temas recorrentes eram a culpa - o sentimento de culpa propriamente dito, e/ou a culpabilização; nesta era frequente um cônjuge atribuir ao outro a responsabilidade pelo problema do filho(a). No grupo percebíamos também a necessidade de saberem qual a causa da dependência e que uma grande 
expectativa de cura era depositada na internação. Nas famílias que já tinham experenciado a internação uma ou várias vezes era evidente a ambivalência entre "ajudar novamente e o largar de mão". Os dependentes químicos na terapia multifamiliar traziam como tema um enorme sentimento de culpa pelo sofrimento causado à família com a sua conduta, em muitos casos agressiva, e uma necessidade enorme de reparação. Estes sentimentos eram explicitados através de palavras e também de expressões e/ou gestos (não verbais) e o arrependimento era demonstrado na sessão multifamiliar, sob forte impacto emocional repercutindo no grupo todo. Estes temas eram trazidos pelas famílias que estavam entre a $1^{\text {a }}$ e a $3^{\mathrm{a}}$ sessão. Quando estavam próximos da alta hospitalar, isto é, entre a $4^{\mathrm{a}}$ e a $6^{\mathrm{a}}$ sessão, o medo da recaída, o receio do convívio familiar e o enfrentamento da realidade eram tratados nas sessōes. Durante o desenvolvimento do processo terapêutico as dificuldades familiares que compartilhavam entre si transformavam-se em questionamentos, reflexões e vivências, nas quais também encontravam possibilidades de mudanças. A partir da temática (dores, temores e perdas) que as famílias de forma muito colaborativa expressavam durante as sessóes, seus membros descobriam e construíam juntos uma rede de apoio e solidariedade, na qual a impotência cedia espaço para um comprometimento com a mudança. Trabalhava-se com o foco em dificuldades da família que poderiam problematizar a recuperação e com as possibilidades de mudanças familiares que se constituiriam protetoras e facilitadoras da recuperação. Durante os anos nos quais foram realizados os grupos multifamiliares, ao término de cada encontro, foi feito um registro pelo terapeuta coordenador do grupo a respeito de dados sociodemográficos dos pacientes e familiares que participaram das sessôes.

Para esse estudo foi considerada adesão ao tratamento quando os sujeitos participaram de, no mínimo, $50 \%$ do processo terapêutico (3 sessôes) ou mais.

\section{PROCEDIMENTO ESTATÍSTICO}

Este registro foi organizado em um banco de dados através do Statistical Package for Social Sciences - SPSS, versão 11.5. Foi realizada estatística descritiva, com medidas de médias e frequências. $\mathrm{Na}$ análise inferencial, foram utilizados o Teste Qui-Quadrado, Teste t de Student para Amostras Independentes, o Teste Exato de Fisher e a Regressão Linear Múltipla, método Stepwise. O nível de significância utilizado foi o de $5 \%$. 


\section{RESULTADOS}

\section{DADOS SOCIODEMOGRÁFICOS}

O grupo de pacientes cujos familiares aderiram à terapia multifamiliar (G1) foi constituído de 427 participantes. No grupo de pacientes cujos familiares não aderiram ao tratamento (G2) havia 244 membros. Os dados sociodemográficos dos sujeitos encontram-se na Tabela 1 (ver anexo).

As substâncias utilizadas pelos pacientes identificados com múltiplas dependências foram, em ordem decrescente: álcool ( $\mathrm{n}=541 ; 80,5 \%)$, cocaína aspirada $(\mathrm{n}=303 ; 45,1 \%)$, maconha $(\mathrm{n}=265 ; 39,4 \%)$, outras substâncias $(\mathrm{n}=160 ; 23,8 \%)$ e cocaína injetável ( $n=64 ; 9,5 \%)$.

Não foi encontrada associação entre a adesão ao tratamento e o tipo de substância utilizada, resultado que está demonstrado na Tabela 2 (ver anexo). Não foi encontrada diferença na adesão no que diz respeito ao fato de ter sido hospitalizado pelo mesmo motivo anteriormente $(\mathrm{p}=0,570)$. Houve diferença, de acordo com o Teste Qui-Quadrado $\left(x^{2}=18,51 ; \mathrm{p}=0,002\right)$, entre a aderência ao tratamento e o grau de parentesco dos familiares que mais participaram dos grupos. Os dados encontram-se demonstrados na Tabela 3 (ver anexo).

Quanto à participação de mais de um familiar e à adesão ao tratamento, de acordo com o Teste Exato de Fisher, foi encontrada associação entre estas variáveis $(\mathrm{p}<0,001)$. A participação de mais de um familiar esteve associada a uma maior adesão. Estes dados encontram-se descritos na Tabela 4 (ver anexo).

Foi utilizada a Regressão Linear Múltipla, método Stepwise para avaliar o modelo que melhor explica a adesão dos familiares ao tratamento multifamiliar. Fizeram parte do modelo as seguintes variáveis: quantidade de familiares $(B=$ $0,369 ; \mathrm{p}<0,001)$; familiar que mais participou $(B=-0,248 ; \mathrm{p}<0,001)$ e idade $(B$ $=0,108 ; \mathrm{p}=0,004)$.

\section{DisCUSSÃO}

Esta pesquisa é um estudo retrospectivo focado nos resultados de uma intervenção breve (seis sessōes), de terapia multifamiliar desenvolvida em uma unidade de dependência química de uma clínica psiquiátrica. Os resultados dessa intervenção demonstraram a importância da inclusão do maior número de familiares desde o início do tratamento. 
A dependência química e problemas relacionais são quase sinônimos, portanto, as famílias quando necessitam internar um familiar o fazem com muito sofrimento e com sentimentos ambivalentes de raiva, dor, fracasso, impotência e um desejo enorme de ajudar. Estudo realizado em Montreal (De Civita, Dobkin \& Robertson, 2000) ilustra essas contradições banalizadas muitas vezes pelo contexto terapêutico que se reduzem a uma tradução no mínimo simplista: "são famílias resistentes". Os autores realizaram um estudo sobre barreiras para adesão de "outros significativos" no tratamento ambulatorial de adultos adictos de um serviço de tratamento canadense. Torna-se visível que eles sentem suas próprias necessidades "ignoradas", como no caso de um familiar que expressou o seguinte: "Quando você está tentando ser parte da solução e você é parte do problema, é difícil apoiar, ou seja, é difícil ser parte da 'solução" (De Civita, Dobkin \& Robertson, 2000: 13).

Quanto maior é o número de familiares participantes do grupo de terapia multifamiliar, melhor é a adesão. Esse achado sugere o engajamento de um maior número de familiares no processo terapêutico, desde o início da internação. Indica que na fase inicial (primeiras internaçōes) a família está sensibilizada e disponível e talvez seja mais viável mobilizá-la para o tratamento. Os comportamentos de cuidados que a presença de uma doença crônica gera tendem a esgotar os membros da rede social (Sluzki, 1997).

Quando se trata de dependência química, qualquer intervenção, mesmo que breve, é melhor que nenhuma e está indicada inclusive para pacientes gravemente comprometidos. Além disso, conforme descrito no Project MATCH Research Group (Copello \& Orford, 2002), a unidade familiar oportuniza adesão mesmo antes de aquele que é o abusador de substância estar completamente motivado para a mudança, conforme demonstrado pelas abordagens "unilaterais".

Percebe-se um equívoco nas famílias quando entendem que o tratamento só pode começar quando o dependente admite sua adição. Quando os pais ou outros significativos enfrentam a dependência química, buscam ajuda e revisam suas crenças, percebem que não são impotentes e que a mudança é viável, independentemente do paciente identificado (o dependente químico). Neste momento a família inicia o tratamento. É fundamental que os serviços especializados em dependência química ofereçam espaços de acolhimento aos familiares, independentemente de o paciente identificado aceitá-lo ou não. $\mathrm{O}$ tratamento deve se iniciar pelo familiar que percebe o problema e está mobilizado para fazer alguma coisa. Este familiar poderá convidar outros e, assim, com uma mobilização diferente da família, começa o tratamento. Esta família poderá encontrar nestes grupos, junto com outras famílias, formas de engajar o dependente químico no processo 
de recuperação. Os grupos de pares, de familiares e/ou multifamiliares são opções menos onerosas que podem tornar o tratamento familiar mais acessível.

Como já citamos anteriormente, os resultados da amostra estudada apontaram que a participação de dois ou mais familiares repercute em uma maior adesão ao tratamento. Esses resultados são corroborados por vários estudos que se referem à importância do trabalho de redes (Ravazzola et al., 1997; Schnitman, 2000; Sluzki, 1997). Para os seres humanos as relaçōes sociais contribuem para dar sentido à vida de seus membros e favorecem uma organização da identidade por intermédio do olhar (e das açôes) dos outros. A rede proporciona uma retroalimentação cotidiana a respeito dos desvios de saúde que favorece os comportamentos corretivos através da associação positiva com cuidados com a saúde.

Para a maior parte dos usuários de substâncias psicoativas, o comportamento aditivo contribui para o isolamento individual, reduzindo sua rede à rede de pares - subcultura da droga (Lopes \& Seadi, 2002). Essa redução da rede em muitos casos é semelhante e simultânea ao processo de isolamento familiar, no qual as rotinas são modificadas, os rituais abolidos e a celebração de datas festivas evitadas (Steinglasss et al., 1987). Sugere-se que um dos caminhos importantes no tratamento da dependência química é incluir o maior número de familiares, desde a família nuclear à família extensa. Outro dado importante sugerido nos resultados é que o engajamento dos familiares deve ser uma meta, principalmente no início do tratamento. Ou seja, quanto mais cedo for a inclusão dos familiares, melhores serão as chances de adesão familiar e de sua coparticipação, funcionando como um fator protetor à tendência das famílias em transformar instituições e terapeutas nos responsáveis pela "cura".

Para chegarem até a internação hospitalar as famílias superaram vários obstáculos e este é só o começo do tratamento. Os cuidados demandados pelo dependente químico em sua recuperação são muitos, o tratamento pós-internação é de longa duração e os familiares ora ficam cansados ora desapontados, e alguns pensam em desistir de seguir em frente. Também há o excesso de otimismo, com o depósito de todas as expectativas na internação. Mas a participação de mais familiares colabora para que mais pessoas estejam informadas sobre o seu papel em cada etapa do tratamento. Uma maior colaboração dos parentes repercute ainda em maior empenho do sistema familiar para que construam novas formas de convívio, onde a droga não pode estar presente.

Conforme vários especialistas em terapia multifamiliar "nas reuniōes multifamiliares, as famílias se convocam para ajudar a solucionar o problema de uma e de todas, gerando-se um verdadeiro efeito de rede" (Ravazzola et al., 1997: 302). O uso da terapia de grupo multifamiliar com famílias de risco 
possibilita a muitas famílias a construção e/ou ampliação da rede social muitas vezes empobrecida pela vergonha associada ao estigma dos serviços psiquiátricos (Sluzki, 1997).

$\mathrm{Na}$ clínica da dependência química, a TGMF possibilita que as famílias acionem uma rede de recursos, significando uma ampliação do foco e a geração de uma rede de possibilidades. Os resultados desse estudo ratificam a importância de os serviços especializados em dependência química adotarem a terapia multifamiliar e/ou de rede como mais uma abordagem terapêutica para o tratamento da dependência química e de toda a sua complexidade.

Neste estudo foi considerada adesão ao tratamento àquelas famílias (G1) que participaram de no mínimo 50\% do processo terapêutico (3 sessōes) ou mais. Os resultados demonstram que entre as mais de 600 internações estudadas a substância psicoativa de maior prevalência é o álcool. Assim, confirmou-se que o consumo de drogas lícitas como o álcool, por exemplo, é superior ao de drogas ilícitas, achado semelhante ao estudo conduzido por Penchansky, Szobot \& Scivoletto (2004).

A amostra apresentou um predomínio de pacientes identificados do sexo masculino, reiterando os dados da literatura que apontam uma maior prevalência deste gênero entre os usuários de substâncias psicoativas (Almeida-Filho et al., 2004).

Da mesma forma, a idade mínima encontrada na amostra, que foi de 14 anos, obteve respaldo na literatura científica, que evidencia o início cada vez mais precoce do uso, abuso e dependência de substâncias psicoativas (Carlini et al., 2005; Penchansky et al., 2004).

Neste estudo os mais jovens foram os P.I. dependentes de inalantes com a idade média de 19,95 anos e os mais velhos os dependentes de medicamentos e de álcool com idades médias de 34,34 e de 35,53 anos, respectivamente. O percurso feito por algumas pessoas, do beber nocivo à dependência do álcool, pode demorar até 30 anos. Como nossa cultura é extremamente permissiva com o consumo de bebidas alcoólicas há uma maior tolerância e, em muitos casos, a busca de tratamento é postergada. Muitos dependentes do álcool são internados em um hospital geral para tratarem somente as complicações clínicas. Não houve diferença na adesão quanto ao sexo, estado civil e idade.

A presença de pais, mães e filhos esteve associada a uma maior adesão ao tratamento, resultado corroborado pelo estudo de Contel e Villas-Boas (1999), no qual a adesão do paciente ao tratamento está relacionada a um maior número de sessões frequentadas pelo familiar. As mulheres, especialmente as mães, foram a presença mais constante, respondendo por $80 \%$ em cada grupo.

A participação dos filhos também esteve associada à adesão ao tratamento. No estudo foram agrupados tanto os filhos menores, quanto os maiores de doze 
anos. Conforme dados da literatura especializada em família (Grandesso, 2000; Penso \& Sudbrack, 2004), sabe-se que quando uma doença crônica surge em uma família existe a possibilidade de que um (ou mais) dos filhos (em geral o mais velho) seja alçado à função de filho parental, ou seja, "o filho que cuida de um ou de ambos os pais".

Os filhos "cuidadores de seus pais" são aqueles que assumem o cuidado dos irmãos menores, buscam seus familiares nos bares ou pagam dívidas de drogas e estão dia e noite vigilantes, abdicando de viverem suas próprias vidas. Entende-se que esse poderia ser o foco de um outro estudo.

No trabalho com a TGMF, famílias com configuraçōes muito diferentes, de nível de instrução e de condições econômicas variadas unem-se no enfrentamento de um problema tão grave como é a dependência de drogas.

\section{ConClusôES}

As pesquisas evidenciam a etiologia multideterminada da dependência química e a família como um desses fatores determinantes.

Existem poucas pesquisas associando a dependência química às intervençôes focadas na família e na rede social, apesar da preocupação da sociedade com o aumento de problemas relacionados ao uso e abuso de substâncias, e consequentemente à existência de muitas famílias envolvidas. Os serviços especializados em dependência química, em sua maioria, não adotam a abordagem familiar como uma prática sistemática.

Sem dúvida, a família tem um papel fundamental como protetora para o uso e abuso de substâncias, mas, quando a dependência química já é um fato, o tratamento, inclusive da família, deve ser adotado sempre e a prática evidencia que o quanto antes melhor. Na medida em que para cada dependente químico esperamse, no mínimo, quatro a cinco pessoas envolvidas, são relevantes estudos que possam contribuir para uma visão ecológica no tratamento da dependência química na qual sua multicausalidade é considerada, inclusive a família.

Nas situações em que o dependente está realizando tratamento hospitalar pela primeira vez na vida, há um sofrimento familiar muito grande e, ao mesmo tempo, muitas expectativas são depositadas na instituição que o recebe, como a de que a internação magicamente resolverá o problema.

A participação de dois ou mais familiares repercute em uma adesão de até $57,9 \%$. Esses resultados sugerem que a participação de um número maior de familiares poderá repercutir em uma maior adesão ao tratamento. 


\section{REFERÊNCIAS BIBLIOGRÁFICAS}

Almeida-Filho, N.; Lessa, I.; Magalhães, L.; Araújo, M. J.; Aquino, E.; Kawachi, I. \& James, S. A. (2004). Alcohol drinking patterns by gender, ethnicity, and social class in Bahia, Brazil. Revista de Saúde Pública, 38 (1), 45-54.

Carlini, E. A.; Galduróz, J. C.; Noto, A. R.; Carlini, C. M.; Oliveira, L. G.; Nappo, S. A.; Moura, Y. G. \& Sanchez, Z. V. D. M. (2005). II levantamento domiciliar sobre o uso de drogas psicotrópicas no Brasil: estudo envolvendo as 108 maiores cidades do país. São Paulo: CEBRID-UNIFESP.

Contel, J. O. B \& Villas-Boas, M. A. (1999). Psicoterapia de grupo de apoio multifamiliar (PGA) em hospital-dia (HD) psiquiátrico. Revista Brasileira de Psiquiatria, 21 (4), 225230.

Copello, A. \& Orford, J. (2002). Addiction and the family: is it time for services to take notice of the evidence? (Editorial) Society for the Study of Addiction to Alcohol and Other Drug Addictions, 97, 1361-1363.

De Civita, M.; Dobkin, P. L. \& Robertson, E. (2000). A study of barriers to the engagement of significant others in adult addiction treatment. Journal of Substance Abuse Treatment, 19 (2), 135-144.

De Micheli, D. \& Formigoni, M. (2001). As razôes para o primeiro uso de drogas e as circunstâncias familiares prevêem os padrōes de uso futuro? Jornal Brasileiro de Dependência Química, 2 (1), 20-30.

Figlie, N. B. \& Pillon, S. C. (2001). Dependência química: novos modelos. In: Focchi, G. A.; Leite, M. C. \& Laranjeira, R. Dependência química: novos modelos de tratamento (pp. 61-75). São Paulo: Roca.

Grandesso, M. A. (2000). Sobre a reconstrução do significado: uma análise epistemológica e hermenêutica da prática clínica. São Paulo: Casa do Psicólogo.

Halpern, S. C. (2001). O abuso de substâncias psicoativas: repercussōes no sistema familiar. Pensando famílias, 3, 120-125.

Jancin, B. (2003). Multifamily therapy holds promise for anxiety. Clinical Psychiatry News, $31(10), 34$.

Laranjeira, R.; Jungerman, F. \& Dunn J. (1998). Drogas: maconha, cocaina e crack. São Paulo: Contextos.

Liddle, H. A.; Dakof, G. A.; Parker, K.; Diamond, G.; Barrett, K. \& Tejeda, M. (2001). Multidimensional family therapy for adolescent drug abuse: results of a randomized clinical trial. American Journal Drug Alcohol Abuse, 27 (4), 651-688. 
Lopes, J. \& Seadi, S. (2002). Avaliação familiar: construindo uma nova maneira de olhar. In: Pulcherio, G.; Bicca, C. \& Silva, F. A. (orgs.). Álcool, outras drogas \& informação: o que cada profissional precisa saber (pp. 61-75). São Paulo: Casa do Psicólogo.

Marques, A. C. P. R. \& Furtado, E. F. (2004). Brief interventions for alcohol related problems. Revista Brasileira de Psiquiatria, 26 (supl. 1), 28-32.

Marques, A. C. P. R. \& Ribeiro, M. (2003). Usuário: abordagem geral. In: Laranjeira, R.; Oliveira, R.; Nobre, M. \& Marques, W. B. Usuários de substâncias psicoativas: abordagem, diagnóstico e tratamento (pp. 12-28). São Paulo: Conselho Regional de Medicina do Estado de São Paulo/Associação Médica Brasileira.

Meyers, J., Apodaca, T., Ficker, S. \& Slenisck, N. (2002). Evidence-Based Approaches for the Treatment of Substance Abusers by Involving Family Members. The Family Journal: Counseling and Therapy for Couples and Families, 10 (3), 281-288.

Miermont, J. \& Molina-Loza, C. (1994). Dicionário de terapias familiares: teorias e práticas. Porto Alegre: Artes Médicas.

Penso, M. \& Sudbrack, M. F. (2004). Involvement in illegal acts and with drugs as possibilities to deal with the role of the parental child. Psicologia USP, 15 (3), 29-54.

Penchansky, F.; Szobot, C. \& Scivoletto, S. (2004). Uso de álcool entre adolescentes: conceitos, características epidemiológicas e fatores etiopatogênicos. Revista Brasileira de Psiquiatria, 26 (supl. 1), 14-17.

Ravazzola, M. C.; Barilari, S. \& Mazieres G. (1997). A família como grupo e o grupo como família. In: Zimerman, D. \& Osório L. C. (orgs.). Como trabalhamos com grupos (pp. 293-304). Porto Alegre: Artes Médicas.

Schenker, M. \& Minayo, M. C. S. (2004). A importância da família no tratamento do uso abusivo de drogas: uma revisão da literatura. Caderno de Saúde Pública, 20 (3), 3-8.

Schnitman, D. F. (2000). Una perspectiva generativa para el trabajo con familias: redes conceptuales y redes de recursos. Pensando famílias, 2, 13-38.

Scivoletto, S. \& Andrade A. D. (2001). Características sócio-demográficas e fatores preditivos no tratamento de adolescentes usuários de drogas. Jornal Brasileiro de Dependência Química, 2 (1), 9-19.

Silva, E. A. (2001). Abordagens familiares. Jornal Brasileiro de Dependência Química, 2 (Supl. 1), 21-24.

Sluzki, C. E. (1997). La red social: frontera de la practica sistemica. São Paulo: Casa do Psicólogo.

Stanton, M. T. \& Todd, D. (1987). Terapia familiar del abuso y adicción a las drogas. Buenos Aires: Gedisa.

Steinglasss, P.; Bennett, L. A; Wolin, S. J. \& Reiss D. (1987). La familia alcoholica. Buenos Aires: Gedisa. 
Sugar, M. (1986). The adolescent in group and family therapy. Nova Iorque: Brunner/Mazel. Thorngren, J. M. \& Kleist D. M. (2002). Multiple Family Group Therapy: An Interpersonal/Postmodern Approach. The Family Journal: Counseling and Therapy for Couples And Families, 10 (2), 167-176.

\section{Notas}

1 Dissertação de mestrado apresentada ao Programa de Pós-Graduação da Faculdade de Psicologia da Pontifícia Universidade Católica do Rio Grande do Sul (PUCRS), como requisito parcial para obtenção do Grau de Mestre em Psicologia Clínica.

\section{AneXo}

TABela 1 - Dados SOCIODEMOGRÁFICOS DA AMOSTRA ( N = 672)

\begin{tabular}{|c|c|c|c|c|}
\hline & & G1 Adesão n ${ }^{\circ}(\%)$ & G2 Não adesão ${ }^{\circ}(\%)$ & $\mathrm{p}$ \\
\hline \multirow[t]{2}{*}{ Sexo } & Masculino & $349(81,9 \%)$ & $196(80,3 \%)$ & $0,340^{* *}$ \\
\hline & Feminino & $77(18 \%)$ & $48(19,7 \%)$ & \\
\hline \multirow[t]{4}{*}{ Est. Civil } & Solteiro & $206(48,9 \%)$ & $130(53,7 \%)$ & $0,376^{*}$ \\
\hline & Casado & $132(31,4 \%)$ & $69(28,5 \%)$ & \\
\hline & Separado & $80(19 \%)$ & $39(16,1 \%)$ & \\
\hline & Viúvo & $3(0,7 \%)$ & $4(1,7 \%)$ & \\
\hline \multirow[t]{4}{*}{ Procedência } & POA e Grande & $325(77,2 \%)$ & $171(70,1 \%)$ & $0,052^{*}$ \\
\hline & POA & $89(21,1 \%)$ & $71(29,1 \%)$ & \\
\hline & Interior & $7(1,7 \%)$ & $2(0,8 \%)$ & \\
\hline & Outros Estados & & & \\
\hline \multirow[t]{2}{*}{ Idade } & & $M=34,41$ & $M=33,11$ & $0,198^{* * *}$ \\
\hline & & $(\mathrm{DP}=12,62)$ & $(\mathrm{DP}=12,44)$ & \\
\hline
\end{tabular}

${ }^{*}$ Qui-quadrado

**Teste Exato de Fisher

*** Teste T de Student para amostras independentes 
Tabela 2 - Distribuição dos PI com Múltiplas DependênCias e Adesão ao Tratamento

\begin{tabular}{|lcccccccc|}
\hline & \multicolumn{2}{c}{ Até 2 sessões } & & \multicolumn{3}{c}{3 sessões ou mais } & & \\
\cline { 2 - 3 } Tipo de substância & $\mathbf{n}$ & $\%$ & & $\mathbf{N}$ & $\%$ & & n & p \\
\hline Álcool & 201 & 82,4 & & 339 & 79,4 & 540 & 0,202 \\
Cocaína Inalada & 115 & 47,1 & & 187 & 43,8 & 302 & 0,225 \\
Maconha & 101 & 41,4 & & 164 & 38,4 & 265 & 0,248 \\
Cocaína injetável & 19 & 7,8 & & 45 & 10,5 & 64 & 0,151 \\
Outra dependência & 54 & 34 & & 105 & 66 & 159 & 0,509 \\
\hline
\end{tabular}

Teste Exato de Fisher

TABela 3 - Adesão aO TRATAMENTO E grau de PARENTESCO DOS FAMILIARES QUE MAIS PARTICIPARAM DOS GRUPOS

\begin{tabular}{|lrrrrrrr|}
\hline & \multicolumn{2}{c}{ Até 2 sessões } & & \multicolumn{2}{c|}{3 sessões ou mais } & \\
\cline { 2 - 3 } Grupo Familiar & \multicolumn{1}{c}{$\mathbf{n}$} & $\%$ & & $\mathbf{n}$ & $\%$ & Total \\
\hline Mãe & 136 & 31,9 & & 57 & 23,5 & 193 \\
Pai & 21 & 4,9 & & 12 & 4,9 & 33 \\
Filho & 20 & 4,7 & & 11 & 4,5 & 31 \\
Companheiro & 116 & 27,2 & & 53 & 21,8 & 169 \\
Irmãos & 21 & 4,9 & & 8 & 3,3 & 29 \\
Mais de 1 familiar & 112 & 26,3 & & 102 & 42 & 214 \\
\hline
\end{tabular}

TABELA 4 - ASSOCIAÇÃO ENTRE O NÚMERO DE FAMILIARES PARTICIPANDO DAS SESSÕES E A ADESÃO AO TRATAMENTO

\begin{tabular}{|lcccccc|}
\hline \multirow{2}{*}{ Familiar } & \multicolumn{4}{c|}{ ADESÃO } \\
\cline { 2 - 3 } & \multicolumn{2}{c}{ Até 2 sessões } & & 3 sessões ou mais & \\
\cline { 2 - 3 } \cline { 6 - 7 } & $\mathbf{n}$ & $\%$ & & N & $\%$ & n \\
\hline Até 1 familiar & 111 & 45,9 & & 77 & 18,1 & 188 \\
Mais de 1 familiar & 131 & 54,1 & & 349 & 81,9 & 480 \\
\hline
\end{tabular}

Recebido em 03 de março de 2008 Aceito para publicação em 15 de junho de2009 